\title{
L'eau au cœur du processus de paix entre Israël et l'OLP Propositions
}

\author{
J. Sironneau \\ Ministère de l'environnement - Direction de l'eau
}

Nous publions ici un article sur un sujet d'actualité. L'auteur y exprime ses opinions sur la situation actuelle du partage de l'eau au Proche-Orient et propose des actions à mettre en cuvre durant le processus de paix entre Israël et l'OLP. Certains peuvent avoir des idées différentes et nous sommes prêts à recevoir leur courrier.

Le Proche-Orient subit une pénurie d'eau structurelle aggravée ces dernières années par des sécheresses récurrentes - notamment celles des années 1989 à 1991 qui ont mis en danger le développement économique et agricole des pays de la région et leur ont fait prendre conscience de la précarité de ressources en eau déjà limitées [1].

En particulier, Israël est situé dans ce que certains auteurs dénomment la «tranche critique» ou «Water stress zone " [2] accusant avec la Jordanie un déficit hydrique d'au moins 300 millions de mètres cubes par an.

La « Déclaration de principe sur des arrangements intérimaires d'autonomie " signée le 9 septembre 1993 entre Israël et l'Organisation de Libération de la Palestine (O.L.P.) aborde bien évidemment le problème de l'eau, s'inscrivant dans le processus des négociations multilatérales israélo-arabes amorcé à Moscou les 28 et 29 janvier 1992 [3] en vue d'aboutir à la paix et dont l'eau constitue l'un des cinq volets.

M. Thomas NAFF en résume parfaitement l'enjeu, pour lequel «Il ne peut y avoir de paix sans régler le problème de l'eau et vice versa (...). C'est l'eau qui déterminera l'avenir des territoires et, au-delà, la paix ou la guerre. Si la crise n'est pas résolue, il en résultera une plus grande probabilité d'un conflit entre la Jordanie et Israël qui entraînerait certainement d'autres pays arabes „[4].

La poursuite et l'issue des négociations actuelles entre Israéliens et Palestiniens sont bien évidemment conditionnées par les garanties d'accès à la ressource en eau que l'O.L.P. serait disposée à consentir à Israël dans l'hypothèse de la constitution d'un état Palestinien.

En effet à juste titre, toujours selon M. Thomas NAFF, " il est inconcevable qu'un gouvernement israélien abandonne jamais une quelconque partie des territoires occupés sans qu'un plan effectif lui assure un accès permanent sur ces territoires à une ressource en quantité suffisante ou à un accès à d'autres sources comparables dans la région [5], telles que probablement le Litani ou l'Auwali au Liban ».
Le contexte régional dans lequel s'inscrit le processus de paix entamé entre Israël et l'O.L.P. demeure un contexte de rareté de la ressource en eau et de croissance démographique qui marque une aggravation prévisible des disparités.

Jusqu'alors, Israël s'est livré à une recherche incessante du contrôle d'une zone hydrologiquement stratégique qui s'est caractérisée par l'échec des plans de partage successifs de la ressource.

La résolution du problème est conditionnée par la conduite conjointe d'avancées politiques et techniques dans un cadre régional dépassant le seul différend israélopalestinien.

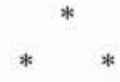

I U UN CONTEXTE RÉGIONAL DE RARETÉ DE LA RESSOURCE EN EAU ET DE CROISSANCE DÉMOGRAPHIQUE MARQUANT UNE AGGRAVATION PRÉVISIBLE DES DISPARITÉS

\subsection{Le déficit hydrique}

Actuellement, Israël utilise d'ores-et-déjà $98 \%$ de ses ressources propres soit 1650 millions de $\mathrm{m}^{3}$ se répartissant entre les nappes souterraines (950), le Jourdain et le lac de Tibériade (600) et les eaux de ruissellement (60 à 100) [6]. - Ses ressources renouvelables théoriques sont estimées par le B.R.G.M. à 2150 millions de $\mathrm{m}^{3}$ soit $501 \mathrm{~m}^{3}$ par an et par habitant en 1985 avec une prévision très forte à la baisse pour 2020 , date à laquelle ces ressources par an et par habitant ne s'élèveraient plus qu'à $327 \mathrm{~m}^{3}$.

Dans la région, seule la Jordanie dispose de ressources inférieures avec seulement 1100 millions/an s'agissant de ses ressources renouvelables théoriques soit $313 \mathrm{~m}^{3}$ par an et par habitant en 1985 et $91 \mathrm{~m}^{3}$ en 2020. 
Tableau 1.

\begin{tabular}{|l|c|c|c|}
\hline Pays & $\begin{array}{c}\text { Ressources } \\
\text { renouvelables } \\
\text { théoriques } \\
\left(\mathrm{Gm} \mathrm{m}^{3} / \mathrm{an}\right)\end{array}$ & $\begin{array}{c}\text { Ressources } \\
\text { renouvelables } \\
\text { théoriques } \\
\text { par tête }(1985)\left(\mathrm{m}^{3} / \mathrm{an} / \mathrm{hab}\right)\end{array}$ & $\begin{array}{c}\text { Ressources } \\
\text { renouvelables } \\
\text { théoriques } \\
\text { par tête }(2020)\left(\mathrm{m}^{3} / \mathrm{an} / \mathrm{hab}\right)\end{array}$ \\
\hline Israël & 2.15 & 501 & 327 \\
Jordanie & 1.10 & 313 & 91 \\
Liban & 4.80 & 1798 & 980 \\
Syrie & 35.50 & 3387 & 1225 \\
Turquie & 203.0 & 4108 & 2340 \\
Egypte & 58.30 & 1238 & 680 \\
Irak & 100.0 & 6390 & 1285 \\
Iran & 117.5 & 2602 & 67 \\
Arabie Saoudite & 2.2 & 191 & 116 \\
EAU & 0.3 & 231 & 0.0 \\
Koweit & 0.0 & 0.0 & 23 \\
Qatar & 0.02 & 62.5 & \\
\hline
\end{tabular}

Source: BRGM Statistiques sur les ressources et les demandes en eau dans le monde (oct. 1989).

L'aggravation de la situation sur une longue période apparaît donc en l'occurrence quasi-exponentielle.

Toutefois, un premier contraste apparaît si l'on compare ces mêmes valeurs caractéristiques avec celles des pays d'«amont» tels que le Liban et plus encore bien évidemment la Turquie, ce qui d'ores-et-déjà pose à terme le problème d'éventuels transferts d'eau.

Avec $501 \mathrm{~m}^{3}$ par an et par habitant, Israël se situe à l'extrême limite du seuil critique retenu par la classification établie par le B.R.G.M. En effet, le seuil de pénurie est atteint avec $1000 \mathrm{~m}^{3}$ d'eau par an et par habitant, étant entendu qu'à 500 la situation devient critique et qu'audessous de 100, il convient de faire appel à des ressources non traditionnelles telles que le dessalement de l'eau de mer ou le recyclage des eaux usées.

Pour sa part, la Banque mondiale retient les seuils de 1000 et $2000 \mathrm{~m}^{3}$ par habitant (« Report 1992 - Development and the Environment).

En Israël actuellement $80 \%$ de la ressource disponible est consacrée à l'agriculture, $15 \%$ aux usages domestiques et $5 \%$ à l'industrie (cf. infra).

- De la même façon, s'agissant des indices d'exploitation le contraste apparaît très nettement entre les pays d' « amont " bénéficiant d'une marge importante d'exploitation non encore mobilisée (indices d'exploitation très faibles inférieurs à $20 \%$ ) et les pays d' «aval » qui se situent à l'extrême limite de l'exploitation possible voire même déjà au-delà. Tel est le cas de la Jordanie, d'lsraël et plus précisément de la Bande de Gaza. [8].

Ainsi pour Israël, le taux de renouvellement de ses ressources est-il de $15 \%$ inférieur à celui de l'accroissement de sa consommation d'eau.

Le problème majeur de ces déficits persistants réside dans leur caractère cumulatif et le fait qu'ils peuvent devenir rapidement irréversibles, tout particulièrement pour les pays riverains du Jourdain. Ainsi, les eaux du lac de Tibériade et du Jourdain sont quasi-inutilisables, spécialement pour l'irrigation, compte tenu de leur très forte teneur en sel encore accrue par la quantité de prélèvements qu'ils subissent.
Bien qu'Israël ait depuis sa fondation en 1948 réalisé des efforts incessants pour accroître ses ressources en eau renouvelables, une surexploitation constante laisse le pays avec un déficit hydrique qui équivaut approximativement à une année d'approvisionnement [9].

A l'heure actuelle, environ $60 \%$ de son approvisionnement en eau provient des ressources souterraines (aquifère côtier surexploité s'étendant du Mont-Carmel à la Bande de Gaza mais surtout aquifère montagneux situé au cœur des Territoires occupés de Cisjordanie ou aquifère de Judée-Samarie, Yarkon-Taninim, allant du Mont-Carmel à Beer Sheba) [10] et environ $80 \%$ de l'eau de l'aquifère montagneux qui s'écoule naturellement vers l'Ouest est captée par les Israéliens.

D'une manière globale, les deux tiers des eaux consommées par Israël chaque année proviennent des Territoires occupés, en particulier de Cisjordanie et $40 \%$ des installations hydrauliques israéliennes sont situées à l'extérieur des frontières d'Israël antérieures à juin 1967 [11]. Mais les installations de captage de l'eau, en particulier souterraine, consommée en Israël, fonctionnent pour l'essentiel à l'intérieur des frontières d'avant 1967 ce qui signifie que techniquement Israël n'aurait en principe pas besoin d'occuper physiquement ces territoires pour pouvoir disposer de l'eau [12].

En 1990, sur une consommation totale de 1750 millions de $\mathrm{m}^{3}, 1162$ sont consacrés à l'agriculture [13], soit environ $80 \%$.

Les principaux problèmes auxquels est confronté Israël sont constitués par la surexploitation des ressources existantes, le manque de capacité de stockage à long terme et des dysfonctionnements dans la gestion de la ressource liés à la concurrence entre trois principales structures: Tahal chargé de la planification de la ressource en eau, Mékorot [14] responsable de la maîtrise d'œuvre et de l'entretien des ouvrages et le Commissariat pour l'eau (Office of the Water Commissioner) dépendant du ministère de l'agriculture et en tant que tel attentif aux groupes de pression agricoles [15]. On notera que Mékorot gère actuellement au moyen de technologies hydrauliques très sophistiquées, environ $70 \%$ de l'eau utilisée dans les Territoires occupés 
et ex-occupés, gestion qui lui a été confiée par une ordonnance militaire de 1982 [16].

1.2. Le problème démographique : l'aggravation due au retour des diasporas israélienne et palestinienne

Même si pour Israël, le taux de croissance annuelle de sa population n'est que d'environ $2 \%$ (contre 3,6 à 3,8\% en Jordanie et en Syrie) [17] incluant la vague d'immigration (750 000 à 1 million de personnes) qui continue à arriver voire à s'amplifier en provenance de l'ex-Union Soviétique, il n'en demeure pas moins que dans l'hypothèse où un tel rythme se poursuivrait la population passerait en Israël de 4,4 à 7 millions et celle des Palestiniens dans les Territoires occupés et ex-occupés de 1,75 à 4,2 millions dès lors que le problème palestinien serait résolu et la diaspora regroupée [18]. En d'autres termes entre 2015 et 2020 la population pour l'ensemble du bassin du Jourdain (si l'on inclut la croissance de la population jordanienne, sur la même période, de 2,7 à 7 millions) pourrait avoisiner les 16 à 18 millions [19] contre 9 millions actuellement.

Dans l'absolu, des taux de croissance démographique supérieurs à 1,5 peuvent avoir des conséquences catastrophiques pour des pays en développement (cas de la future entité palestinienne en particulier), les confrontant à l'impossibilité d'approvisionner en eau leurs habitants en quantité suffisante, et bloquant de ce fait le développement économique et sociale.

Le Conseil économique et social des Nations Unies s'en est inquiété en soulignant que "le fossé entre des ressources aquifêres limitées et la rapide augmentation de la population (...) s'élargit de façon inquiétante et pourrait mettre en danger la sécurité en matière d'eau dans cette région [20]».

Pour le seul Israël, cela signifie qu'il devrait trouver avant l'an 2000, 800 millions de $\mathrm{m}^{3}$ supplémentaires s'ajoutant aux 1700 millions actuellement exploités, pour faire face aux conséquences de l'immigration.

Du côté palestinien, la Bande de Gaza offre un condensé des problèmes qui se posent dans la région.

1.3. La Bande de Gaza, condensé des problèmes quantitatif et qualitatif

La surpopulation de ce territoire [21], précédemment occupé par les Israéliens et devenu autonome, entraîne en effet la surexploitation de l'aquifère côtier [22] ne communiquant que peu avec les autres nappes souterraines

Tableau 2. - Indices d'exploitation.

\begin{tabular}{|c|c|c|c|c|c|c|c|c|c|}
\hline \multirow[b]{3}{*}{$\begin{array}{l}\text { Pays et } \\
\text { Territoires }\end{array}$} & \multicolumn{4}{|c|}{$\begin{array}{l}\text { Ressources renouvelables } \\
\text { (moyenne annuelle) } \\
\text { (mio } \mathrm{m}^{3} / \mathrm{an} \text { ) }\end{array}$} & \multicolumn{4}{|c|}{$\begin{array}{l}\text { Prélèvements } \\
\left.\text { (mio } \mathrm{m}^{3} / \mathrm{an}\right)\end{array}$} & \multirow{3}{*}{$\begin{array}{c}\text { Indices } \\
\text { d'exploit } \\
(2):(1) \\
\%\end{array}$} \\
\hline & \multicolumn{2}{|c|}{ Ressources internes } & \multicolumn{2}{|c|}{ Ressources externes } & \multirow{2}{*}{$\begin{array}{c}\begin{array}{c}\text { Ressources } \\
\text { totales }\end{array} \\
(1)\end{array}$} & \multirow[b]{2}{*}{ Surfaces } & \multirow[b]{2}{*}{ Souterr. } & \multirow{2}{*}{$\begin{array}{c}\begin{array}{c}\text { Prélèvement } \\
\text { totaux }\end{array} \\
\text { (2) }\end{array}$} & \\
\hline & Surfaces & Souterr. & Surfaces & Souterr. & & & & & \\
\hline $\begin{array}{l}\text { Syrie } \\
\text { Jordanie } \\
\text { Cisjordanie } \\
\text { Israël } \\
\text { Gaza } \\
\text { Liban }\end{array}$ & $\begin{array}{c}4324 \\
156 \\
45 \\
200 \\
\in \\
1750\end{array}$ & $\begin{array}{c}3609 \\
356 \\
580 \\
800 \\
25 \\
3195\end{array}$ & $\begin{array}{c}28000 \\
412 \\
0 \\
400 \\
15 \\
E\end{array}$ & $\begin{array}{l}1200 \\
\bar{\epsilon} \\
270 \\
20 \\
E\end{array}$ & $\begin{array}{c}37430 \\
912 \\
630 \\
1700 \\
60 \\
4945\end{array}$ & $\begin{array}{c}2900 \\
336 \\
375 \\
600 \\
\in \\
650\end{array}$ & $\begin{array}{c}2300 \\
333 \\
237 \\
1190 \\
100 / 130 \\
210\end{array}$ & $\begin{array}{c}5219 \\
669 \\
612 \\
1790 \\
100 / 130 \\
860\end{array}$ & $\begin{array}{c}14 \\
73.5 \\
97 \\
105 \\
160 / 200 \\
17\end{array}$ \\
\hline
\end{tabular}

Source: BRGM-Etat des lieux du Secteur de l'eau au Moyen-Orient (Juin 1992).

Tableau 4. - Accroissement de population au Moyen-Orient.

\begin{tabular}{|l|c|c|c|c|}
\hline Country & $\begin{array}{c}1986 \text { Population } \\
\text { (in millions) }\end{array}$ & Crude Birth Rate & $\begin{array}{c}\text { Natural } \\
\text { Increase }\end{array}$ & $\begin{array}{c}\text { Estimated Population in 2000 } \\
\text { (in millions) }\end{array}$ \\
\hline Lebanon & 2.7 & 29 & 2.1 & 3.6 \\
Jordan & 3.7 & 44 & 3.7 & 6.4 \\
Israël & 4.2 & 23 & 1.6 & 5.3 \\
Syria & 10.5 & 17 & 3.8 & 24.2 \\
Iraq & 16.0 & 46 & 3.3 & 34.2 \\
Sudan & 22.9 & 37 & 2.6 & 71.2 \\
Egypt & 50.5 & 35 & 2.5 & 69.7 \\
Turkey & 52.4 & 16 & 0.7 & 268.0 \\
\hline U.S. & 241.0 & 27 & 1.7 & $6,157.0$ \\
\hline World & $4,942.0$ & & \\
\hline
\end{tabular}

Source: World Population Data Sheet (Washington, D.C.: The Population Reference Bureau. Inc., 1986).

* Crude birth rate is the number of births per 1.000 persons.

** Natural increase is the annual percentage increase in population. 


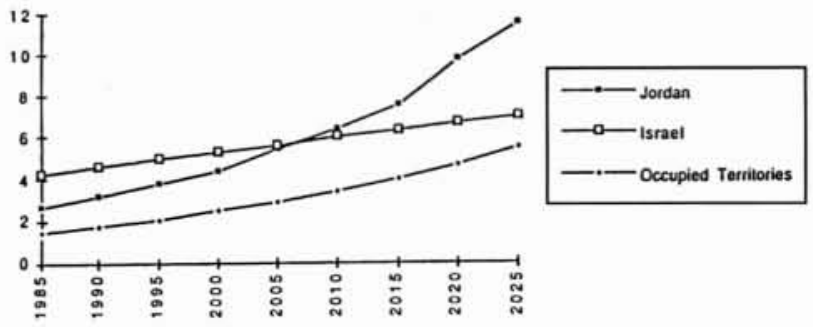

1. Accroissement de la population pour la Jordanie, l'Israël et les territoires occupés (population en millions) source : M. T. Naff.

d'Israël. Il s'ensuit d'importantes et croissantes intrusions d'eau de mer [23], la situation ayant été aggravée par les pompages excessifs pratiqués notamment lors de la période d'occupation par l'Egypte, ainsi qu'une situation alarmante sur le plan de l'hygiène génératrice d'une très importante pollution.

1.4. Concurrence et disparités dans les usages de l'eau entre Israéliens et Palestiniens

Le problème se pose de façon encore plus cruciale lorsque la concurrence entre usages est le fait de systèmes économiques et sociaux disparates.

Dans les Territoires occupés de Cisjordanie, la consommation moyenne journalière pour les usages domestiques est de 70 litres pour un Palestinien, contre une consom- mation de niveau occidental de 260 litres pour un Israélien, soit presque quatre fois plus.

La disparité des consommations d'eau entre les deux communautés ne va pas sans aggraver les difficultés tant au niveau social que psychologique, ceci d'autant qu'il est admis qu'environ $90 \%$ de l'eau extraite de la Cisjordanie est utilisée pour la consommation d'Israël alors que les Palestiniens ne bénéficient que de $10 \%$ de ce volume [24]. Les pratiques discriminatoires entre colons juifs et agriculteurs palestiniens ( $\mathrm{cf}$. infra) ne font qu'amplifier le phénomène.

II LA RECHERCHE INCESSANTE PAR ISRAËL DU CONTRÔLE D'UNE ZONE HYDROLOGIQUEMENT STRATÉGIQUE

2.1. L'échec des plans successifs de partage de la ressource

A l'issue de la «Déclaration BALFOUR » en 1917. WEIZMANN, le président de l'Organisation sioniste mondiale demande lors de la conférence de Paris (1919) que les frontières de la Palestine soient déterminées à partir de considérations hydrauliques et englobant notamment les sources du Jourdain et du Litani, le Golan ainsi que le Yarmouk.

Dès l'instauration du mandat britannique sur la Palestine en 1922, l'Agence juive constitue une commission technique spécialisée afin d'étudier les possibilités d'utilisation

Tableau 3. - Les ressources en eau douce du Moyen-Orient (en $\mathrm{m}^{3}$ par personne et par an)

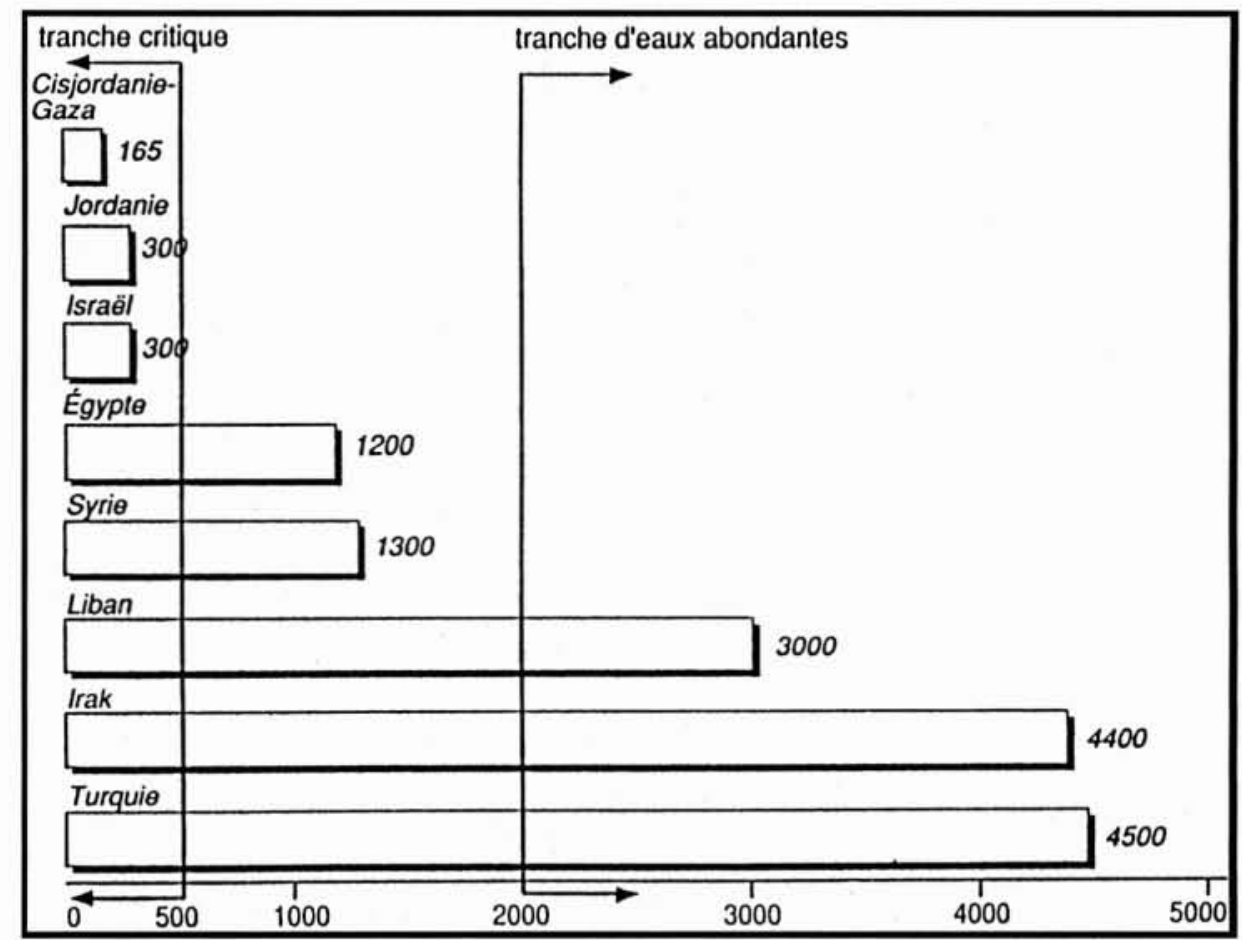

Source : Revue Monde arabe-Maghreb-Machrek n 138, oct.-déc. 1992, p. 34. 
de l'eau et l'irrigation des terres arides, les habitants Arabes de la région élaborant de leur côté leurs propres plans.

Il s'ensuit une succession de plans [25].

1) La concession RUTENBERG (1926): concession hydroélectrique autorisant les juifs de Palestine et eux seuls à utiliser l'eau du Jourdain et du Yarmouk [26] ;

2) Le plan IONIDES (1939) : irrigation des terres à l'est du Jourdain à partir du stockage de l'eau du Yarmouk dans le lac deTibériade et sa dérivation par le canal de East Ghor [27] :

3) Le plan WALTERCLAY-LOWDERMILK (1944): irrigation de la vallée du Jourdain, dérivation des eaux du Jourdain et du Yarmouk en direction du Néguev et développement de l'énergie hydraulique au moyen de la construction d'un canal reliant la Méditerranée à la Mer Morte, sous l'égide d'une autorité gestionnaire ad hoc [28] ;

4) Le plan HAYS (1948) : dérivation de la moitié du débit du Yarmouk vers le lac de Tibériade et vraisemblablement du Litani vers les implantations juives de Palestine ;

5) Le plan MAC DONALD (1951) : utilisation du Jourdain exclusivement en vue de l'irrigation de ses deux rives (construction de canaux parallèles);

6) Le plan JOHNSTON (1953): synthèse des plans LOWDERMILK et HAYS, tentative la plus significative qui ait été faite dans le sens d'un règlement, prévoyait des quotas de répartition dans le bassin du Jourdain. Ce plan n'a toutefois pu être appliqué, bien qu'accepté dans son principe par les principaux protagonistes, du fait en particulier des tensions politiques régionales.

Même si ces plans excédaient par leur objectif et leurs ambitions le cadre strict de la Palestine, celle-ci n'en est pas moins demeurée au cœur de leurs préoccupations.

A telle enseigne que la Guerre des Six Jours (1967) allait permettre à Israël en conquérant la Cisjordanie de résoudre l'un des problèmes de la sécurité d'approvisionnement en eau qu'aucun de ces plans n'avait réussi à résoudre.

2.2. La ressource en eau et la sécurité d'approvisionnement, préoccupation sous-jacente des conquêtes territoriales successives réalisées par Israël

Les conquêtes territoriales successives (Cisjordanie et Golan en juin 1967 au détriment respectif de la Jordanie et de la Syrie) ont permis à Israël de garantir de façon significative sa sécurité d'approvisionnement en eau.

En effet, tout en mettant fin aux projets hydrauliques des Arabes, la Guerre des Six Jours a été pour Israël l'occasion de se rendre maître de la totalité de la vallée du Jourdain jusqu'à la Mer Morte mais surtout de l'aquifère montagneux de Judée et de Samarie.

Cet aquifère d'une importance exceptionnelle dans une zone aride et dont l'aire de recharge se situe dans le sous-sol des Territoires occupés de Cisjordanie est exploité grâce à des puits artésiens de grande profondeur.

L'O.L.P. d'ailleurs ne s'est pas trompée sur les objectifs « hydrostratégiques » d'Israël puisque dès la conquête territoriale effectuée, elle s'en est prise par une série d'attaques directement aux installations hydrauliques israéliennes.

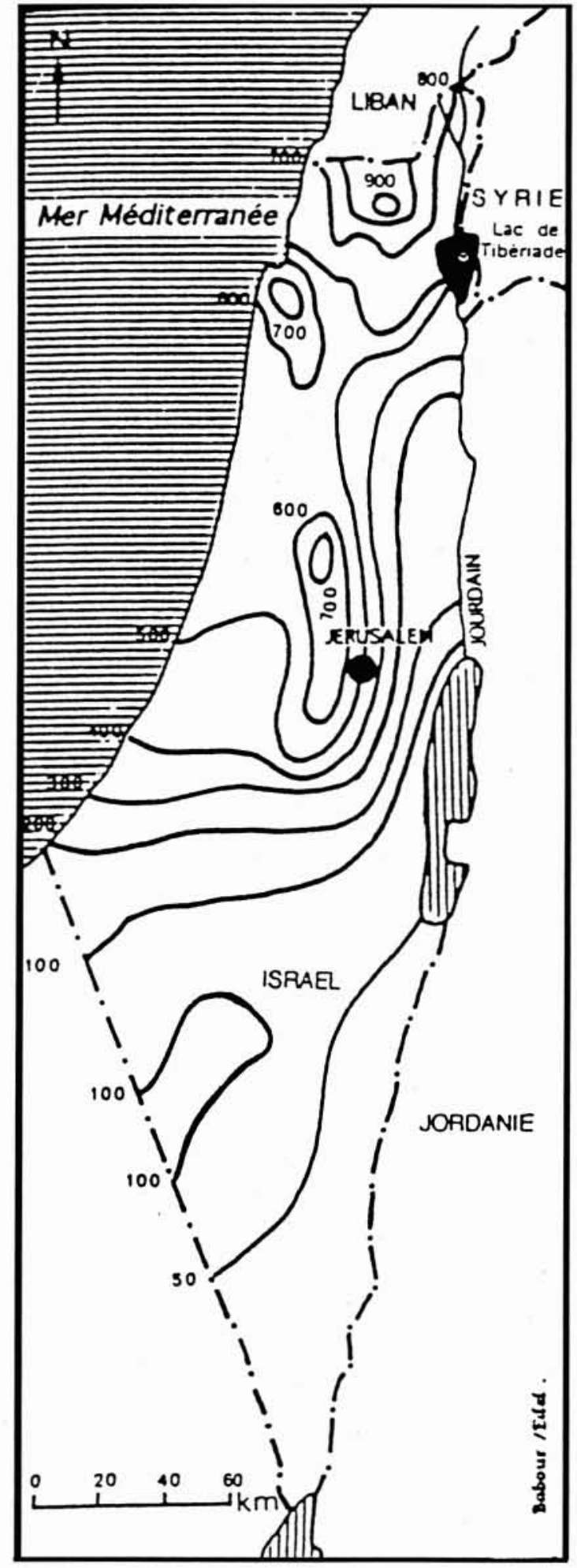

2. Moyennes pluviométriques en Israël et territoires occupés (source : Habib Ayeb.)

Israël accroît l'empire de son « Heartland » hydrologique successivement par la conquête du plateau du Golan, duquel sourd l'un des cours d'eau (le Banias) alimentant le lac de Tibériade (lac Kinneret) en 1982, lors de l'opération «Paix en Galilée ». Cette opération lui permet no- 


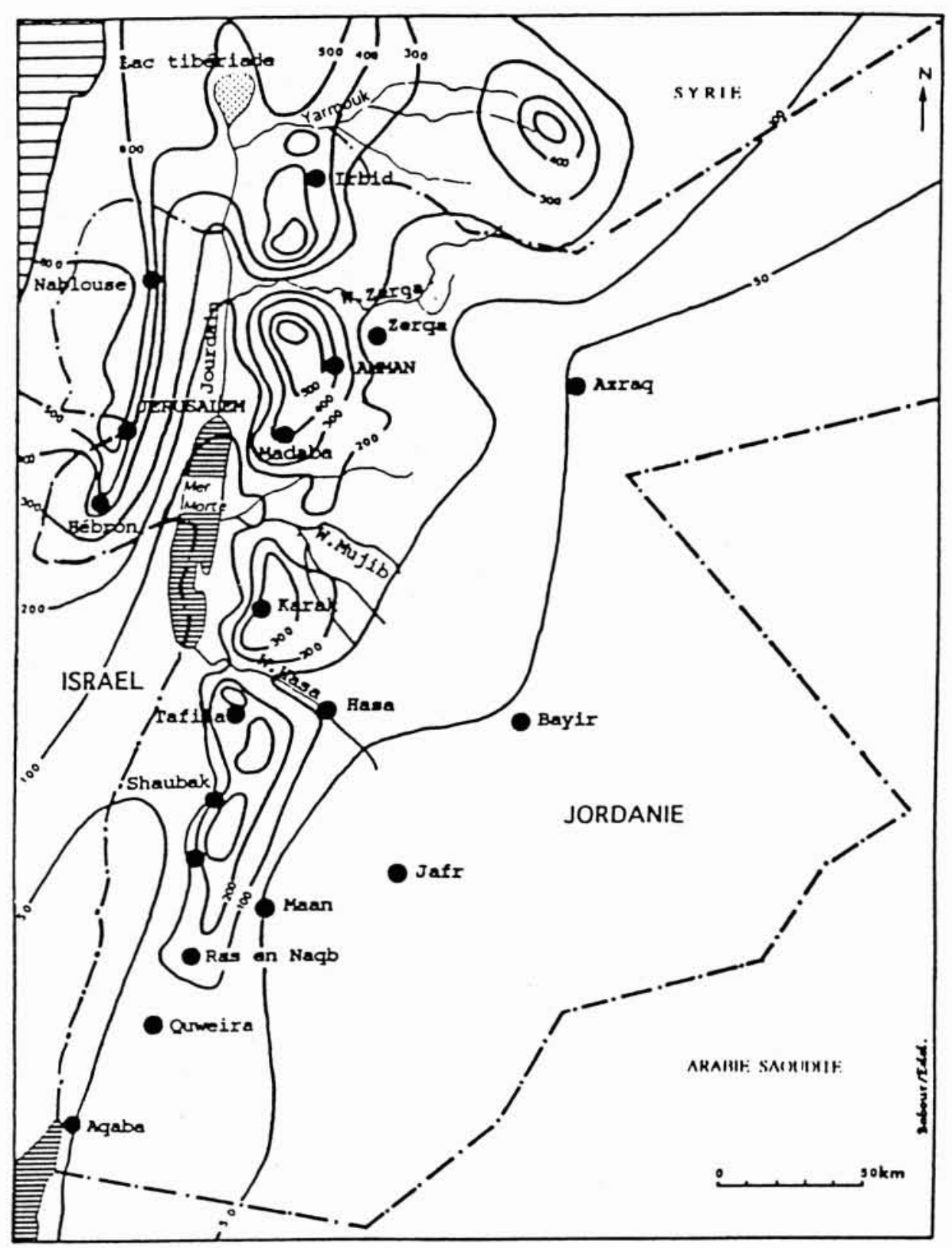

3. Moyennes pluviométriques dans le bassin du Jourdain (source : Habib Ayeb).

tamment de parachever l'œuvre entreprise en s'assurant du contrôle des trois cours d'eau alimentant directement le même lac à savoir le Dan, le Hasbani et le Banias luimême. Ces deux derniers cours d'eau contribuent en effet pour un tiers au débit du Jourdain. En outre, la maîtrise du Golan permet aux Israéliens la libre et entière disposition du lac de Tibériade qui fournit environ le quart de la quantité d'eau consommée par Israël [29].

Enfin, il n'est pas exclu, malgré des difficultés techniques liées en partie à la topographie, qu'Israël dérive à l'heure actuelle grâce à un tunnel une partie des eaux du Litani vers le bassin du Jourdain.

Certains estiment en effet que le maintien des Israéliens dans la partie méridionale du Liban s'expliquerait moins par des considérations purement politiques et stratégiques que par des considérations hydrauliques [30].
Ainsi, le problème israélo-palestinien doit-il être replacé dans son contexte régional.

2.3. Les craintes réciproques des Palestiniens et des Israéliens

Le problème de l'eau apparaît bien évidemment indissociable des droits respectifs des deux communautés tout particulièrement sur l'aquifère montagneux de JudéeSamarie.

En effet, les Palestiniens ont toujours craint jusqu'à maintenant que celui-ci ne soit de plus en plus sollicité par les Israéliens pour satisfaire aux besoins liés à l'implantation de nouvelles colonies du fait de l'immigration massive de Juifs en provenance notamment de l'ex-U.R.S.S. 


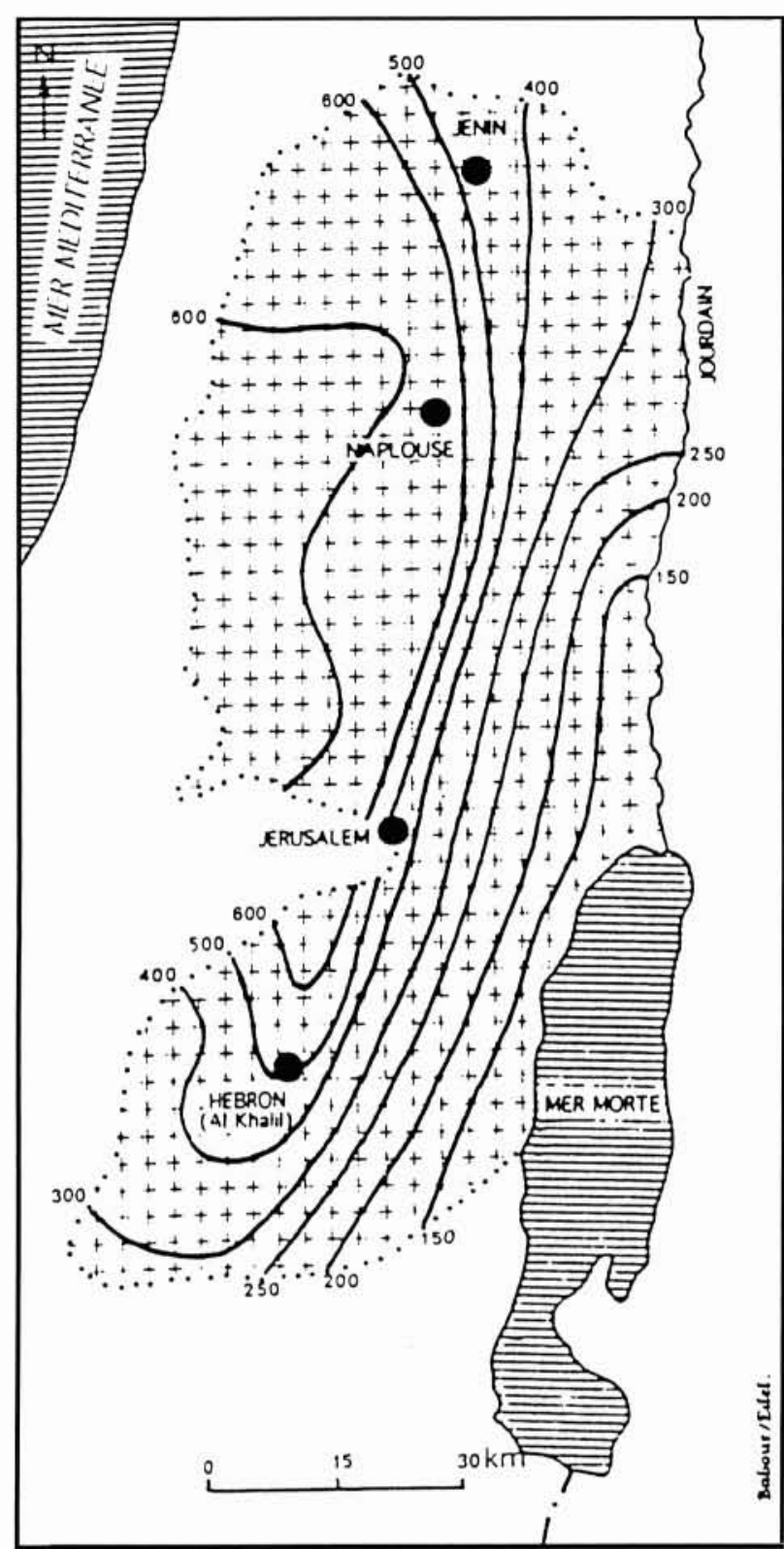

4. Moyennes pluviométriques en Cisjordanie (source : Habib Ayeb).

Ils revendiquent pour leur usage propre cet aquifère, qui selon eux est d'ores-et-déjà surexploité. Ceci aurait provoqué dans certains cas l'assèchement de leurs points d'eau, les rendant ainsi dépendants des Israéliens pour leur approvisionnement en eau.

De son côté. Israël excipe des droits historiques fondés sur l'antériorité de l'usage et nourrit des craintes analogues au cas où les Palestiniens acquériraient leur complète indépendance et favoriseraient une immigration de leur diaspora.

A la réduction quantitative estimée à environ 300 millions de $\mathrm{m}^{3}$ risquant de priver d'eau potable près de 3 millions de personnes, les Israéliens s'inquiètent, même dans l'hypothèse de la conclusion d'un accord équitable, de l'éventualité de rejets mal contrôlés (eaux résiduaires domestiques en particulier) ou d'une importante pollution d'origine agricole qui rendraient, dans la partie aval
Tableau 5. - Les débits du Jourdain selon Naff et Matson

\begin{tabular}{|c|c|c|c|c|}
\hline \multirow[t]{2}{*}{ Sources } & \multirow[t]{2}{*}{ Pays } & \multicolumn{3}{|c|}{ Débits $\left(\mathrm{Mm}^{3}\right)$} \\
\hline & & Apports & Pertes & Total \\
\hline 1. Haut Jourdain & & & & \\
\hline a) Dan & Israël & 245 & & \\
\hline b) Hasbani & Liban & 138 & & \\
\hline c) Banias & Syrie & 121 & & \\
\hline $\begin{array}{l}\text { 2. Jourdain à } \\
\text { Houleh }\end{array}$ & & & & 504 \\
\hline $\begin{array}{l}\text { 3. Irrigation dans } \\
\text { la vallée de Houleh }\end{array}$ & Israël & & -100 & \\
\hline 4. Cours d'eau lo- & Israël & 140 & & \\
\hline $\begin{array}{l}\text { Caux a JISr Banat } \\
\text { Yaakoub }\end{array}$ & & & & \\
\hline $\begin{array}{l}\text { 5. Débit à l'entrée } \\
\text { du lac de Tibériade }\end{array}$ & & & & 544 \\
\hline $\begin{array}{l}\text { 6. Dans le lac de } \\
\text { Tibériade }\end{array}$ & & & & \\
\hline $\begin{array}{l}\text { a) Sources loca- } \\
\text { les }\end{array}$ & $\begin{array}{l}\text { Israël/ } \\
\text { Syrie }\end{array}$ & 70 & & \\
\hline $\begin{array}{l}\text { b) Pluies sur le } \\
\text { lac }\end{array}$ & Israël & 65 & & \\
\hline c) Sources dans & Israël & 65 & & \\
\hline 7. Evaporation & Israël & & 270 & \\
\hline $\begin{array}{l}\text { riade } \\
\text { riac de Mbe- }\end{array}$ & & & & \\
\hline $\begin{array}{l}\text { 8. Ecoulements } \\
\text { vers le bas Jour- } \\
\text { dain }\end{array}$ & & & & 474 \\
\hline 9. Yarmouk & $\begin{array}{l}\text { Syrie/ } \\
\text { Jord }\end{array}$ & 492 & & 966 \\
\hline $\begin{array}{l}\text { 10. Wadis et sour- } \\
\text { ces dans le ghor }\end{array}$ & $\begin{array}{l}\text { Jord./ } \\
\text { Israël }\end{array}$ & 505 & & 1471 \\
\hline
\end{tabular}

Source: Smith (1966), as revised from Main Plan and Hydrological Year-Book of Israel (1946-1960) cité par M. Habib AYEB.

d'écoulement de la nappe en Israël, les eaux de l'aquifère impropres à la consommation humaine.

2.4. Jusqu'alors, une répartition inéquitable de l'eau dans les Territoires occupés

La politique menée par Israël dans les Territoires occupés de Cisjordanie a été de soumettre à restriction les utilisateurs Palestiniens alors qu'il favorisait ses propres colons.

Une ordonnance militaire $\mathrm{n}^{\circ} 158$ du 30 octobre 1967 dispose en effet dans son article 4 (a) qu' "Il est interdit à quiconque de mettre en auvre ou de détenir des installations hydrauliques sans avoir préalablement obtenu une autorisation auprès du commandement militaire [31]."

Une autre ordonnance militaire $\mathrm{n}^{\circ} 92 / 7$ de juin 1967 prévoyait déjà une impressionnante série de restrictions : - interdiction de forer de nouveaux puits sans l'autorisation préalable des autorités militaires (depuis 1967, 34 autorisations seulement ont été accordées et encore toutes l'ont-elles été à des fins domestiques à l'exclusion des fins agricoles ou industrielles) ; 


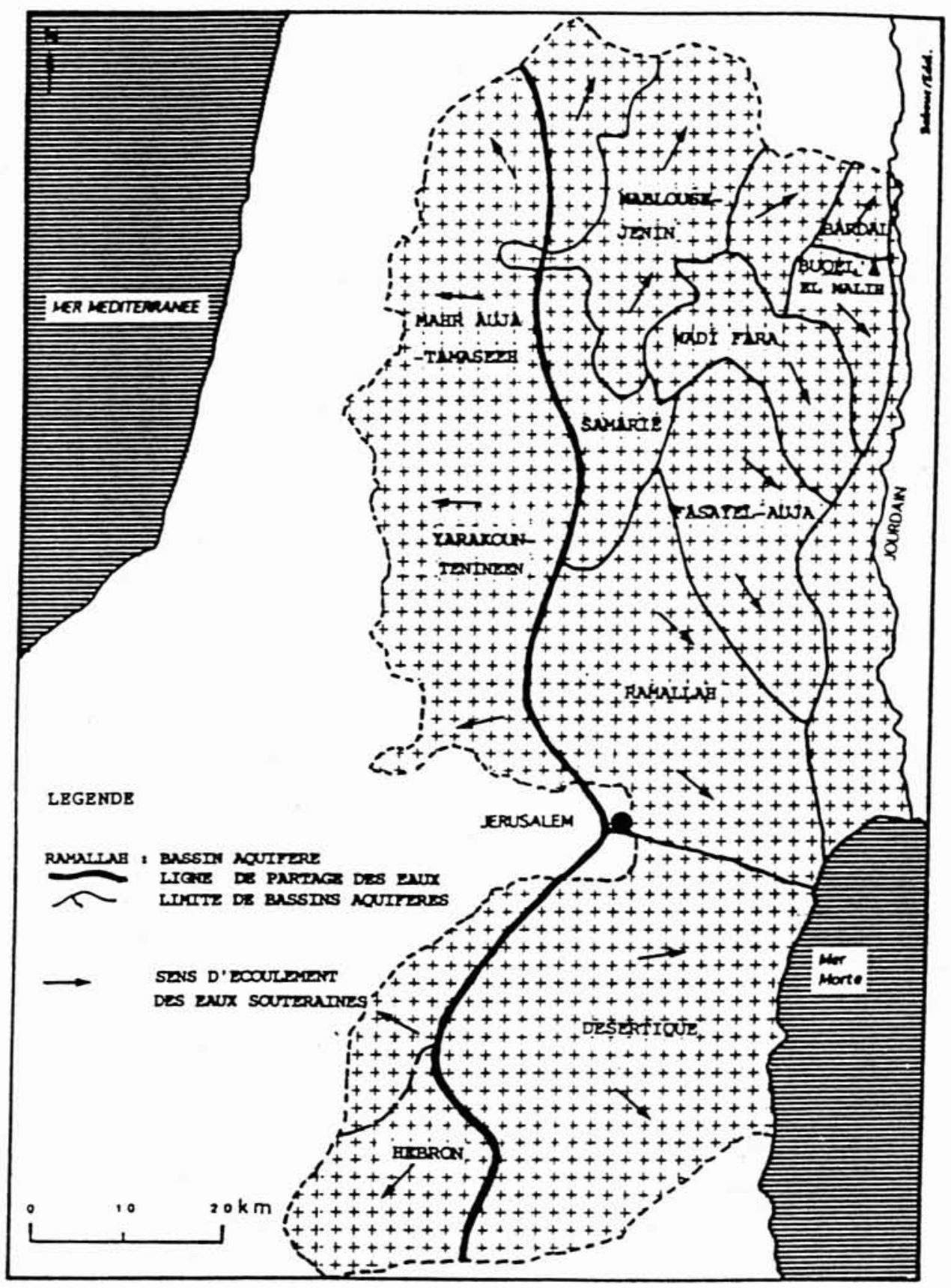

5. Les eaux souterraines en Cisjordanie (source: Habib Ayeb).

- fixation de quotas de prélèvements et mise en place de mécanismes pour contrôler l'utilisation faite de l'eau par les Palestiniens (le dépassement des quotas est sévèrement sanctionné par des amendes);

- expropriation de puits et de sources appartenant à des Palestiniens « absents »;

- interdiction faite aux agriculteurs palestiniens d'irriguer après 16 heures (période traditionnelle pour ce faire).

En outre, la facturation de l'eau dans les Territoires occupés est effectuée de façon identique à la facturation établie en Israël [32], sans tenir compte de la différence de niveau de vie entre les deux communautés.
Enfin, les Palestiniens ont été exclus du bénéfice des subventions accordées aux irrigants israéliens, de sorte que des agriculteurs Palestiniens paient l'eau destinée à l'irrigation au même tarif que celui perçu auprès des Israéliens pour l'eau potable.

D'aucuns estiment que de telles pratiques discriminatoires n'avaient pas pour autre objectif que de contraindre les Palestiniens à quitter ces territoires [33].

Concomitamment, Israël a mené une politique d'implantation de "colonies de peuplement " en particulier en Cisjordanie et au Golan c'est-à-dire dans les zones les mieux dotées en ressources hydriques [34]. 
Le point d'application majeure de cette stratégie de peuplement s'est révélé être la région du Ghor en Cisjordanie qui présente la double caractéristique d'offrir un grand nombre de sources et de contrôler l'ensemble des points de passage entre la Jordanie et la Cisjordanie.

\section{LA CONDUITE CONJOINTE D'AVAN- CÉES POLITIQUES ET TECHNIQUES DANS UN CADRE RÉGIONAL DÉ- PASSANT LE SEUL DIFFÉREND ISRAÉLO-PALESTINIEN}

\subsection{Le règlement du différend israélo-palestinien dans le domaine de l'eau suppose au préalable une avancée diplomatique significative qui est en cours de réalisation}

La reconnaissance mutuelle d'Israël et de l'O.L.P. concrétisée par la « Déclaration de principes sur des arrangements intérimaires d'autonomie" signée le 9 septembre 1993 constitue l'amorce du règlement israélo-palestinien.

$\mathrm{La}$ «déclaration de principes » comporte un important volet consacré, d'une part à l'environnement [35], d'autre part et surtout à l'eau.

En effet, les deux parties conviennent en particulier, dans le cadre d'un protocole de coopération, d'établir un comité de suivi pour la coopération économique qui doit se concentrer notamment sur :

1) La coopération dans le domaine de l'eau, comprenant un programme de développement des ressources hydraulique préparé par des experts des deux parties, qui devra spécifier aussi les modalités de coopération dans la gestion des ressources aquifères en Cisjordanie et dans la Bande de Gaza et faire des propositions d'études de projet sur les droits en eau de chaque partie aussi bien que pour l'utilisation équitable des ressources communes au cours de la phase intérimaire et après ;

2) Un plan de protection de l'environnement prévoyant des mesures conjointes et/ou coordonnées dans ce domaine.

Des programmes de développement régional doivent également être mis en place comportant en particulier :

1) Un programme de développement de l'infrastructure (eau, électricité etc...) ;

2) Un projet régional de dessalement et d'autres projets de développement de ressources hydrauliques.

Le «Conseil», autorité intérimaire palestinienne élue, est chargé d'établir pour la mise en œuvre de ces actions, notamment une autorité palestinienne pour l'eau ou toute autre structure préalablement convenue (articles $\mathrm{I}^{\text {er }}$ et VII.4).

Le processus ainsi engagé de l'autonomie peut laisser présager à terme la constitution d'un Etat palestinien indépendant mais dont la viabilité dépend essentiellement du fait qu'il détiendra des ressources en eau en quantité et qualité suffisantes. D'aucuns estiment que les $1080 \mathrm{mil}-$ lions de $\mathrm{m}^{3}$ de ressource renouvelable annuelle en eau [36] permettraient de subvenir aux besoins d'environ 7.5 millions d'habitants palestiniens (6,7 millions en 2010). Ainsi «l'Etat palestinien aurait des capacités hydrauliques suffisantes pour le premier quart du siècle de son existence et (donc) le facteur hydraulique ne constitue pas un obs- tacle à son édification " [37]. En effet même avec un taux élevé d'évaporation et de déperdition, la Cisjordannie conserve un solde positif global de 836 millions de $\mathrm{m}^{3}$ [37-1].

3.2. Les solutions de partage fondées sur les règles d'Helsinki, d'utilisation raisonnable de la ressource, devraient permettre de surmonter des divergences juridico-religieuses

\subsubsection{Des divergences sémantiques demeurent}

Au-delà du caractère sacré attribué à l'eau aussi bien par le judaïsme que par l'Islam, la législation israélienne considère désormais l'eau comme propriété de l'Etat alors que pour la Charia [38], l'eau, don de Dieu, ne saurait ni faire l'objet d'une quelconque propriété, fût-elle étatique, ni être soumise à aucun contrôle.

3.2.2. Le nécessaire recours aux principes généraux émergeant du droit international de l'eau en gestation

Dès 1966, l'Association du droit international adopte à Helsinki des «Règles » proposant un certain nombre de concepts au nombre desquels celui de l'unité de gestion de la ressource en eau consistant à gérer, par exemple un cours d'eau, de façon globale par l'intégration des aspects quantitatifs et qualitatifs indépendamment des frontières qu'il traverse [39].

La Conférence internationale sur l'eau et l'environnement (Dublin janvier 1992) [40] ainsi que la déclaration de Rio sur l'environnement et le développement [41] mettent l'accent sur la mise en valeur et la gestion intégrées de ressources en eau à l'échelon du bassin dans leur dimension multisectorielle d'utilisation, en demandant aux Etats d'adopter une approche globale qui concilie développement des économies et des sociétés humaines et préservation des écosystèmes naturels dont dépend notre survie. L'approche doit respecter les écosystèmes en considérant solidairement l'ensemble du bassin versant et des nappes souterraines: pour les bassins partagés et les nappes transfrontalières une coopération s'impose [42].

Depuis une vingtaine d'années sous l'égide de l'Assemblée générale des Nations Unies (Résolution 2669 - XXV - du 8 décembre 1970), la Commission du droit international (C.D.I.) élabore un projet de convention pour les utilisateurs des cours d'eau internationaux à des fins autres que la navigation qui, reprenant pour l'essentiel des règles d'Helsinki, reconnaît aux Etats riverains le droit d'utiliser une part "raisonnable et équitable " des eaux mais avec l'obligation faite de ne pas causer de dommages appréciables aux autres Etats riverains (articles 5 à 7).

L'exercice de la souveraineté d'un Etat au droit des eaux coulant sur son territoire ne saurait priver les autres Etats riverains du même droit car l'eau est une ressource naturelle partagée (Res comunis omnium).

Le pendant existe pour les eaux souterraines transfrontalières avec le projet de traité dit de «BELLAGIO » [43] qui applique des règles analogues, à savoir "l'unité de gestion », la "communauté d'intérêts ", l'utilisation optimale et la conservation sur une base raisonnable et équitable (article 2 ), le recours possible à une 
commission internationale autorisée à déclarer des zones de protection, d'alerte à la sécheresse, à élaborer des plans d'urgence, l'échange réciproque d'informations résultant d'inventaires qualitatifs et quantitatifs.

Le projet de traité prévoit également la mise en œuvre de plans de gestion de la ressource très complets dans les zones de protection ainsi que des plans « sécheresse ».

Enfin, un mécanisme très sophistiqué de résolution des différends comportant plusieurs degrés, accorde d'importants pouvoirs à la commission précitée. En effet, dans un premier temps, celle-ci s'efforce de résoudre le différend. Si elle n'y parvient pas, le ou les gouvernements demandent un rapport à une commission d'enquête. La commission poursuit alors ses efforts de règlement sur la base du rapport et si un accord s'avère impossible, les gouvernements s'engagent à avoir recours à tous procédés de médiation, conciliation, arbitrage, à la Cour internationale de justice ou à tout autre moyen adéquat (articles 15 et 16).

Le 17 mars 1992 a été signée à Helsinki la Convention sur la protection et l'utilisation des cours d'eau transfrontières et des lacs internationaux élaborée sous l'égide de la Commission économique pour l'Europe des Nations-Unis. Moins ambitieux que le précédent projet, cette convention-cadre (à laquelle peuvent seulement adhérer les pays-membres de la CEE-NU y ayant un observateur) contient quelques principes généraux sur la gestion des ressources en eau (principe de précaution, principe pollueur-payeur), des principes de concertation et de coopération («usage raisonnable et équitable», gestion patrimoniale des eaux transfrontières visant à ne pas compromettre les usages des générations futures (cl. not. art. $2.2 \mathrm{c}$ et $2.5 \mathrm{a}$ b et c) ainsi que des clauses destinées à servir de référence à des accords de cours d'eau (partie II).

Même si les projets de traités et la convention signée n'envisagent pas toutes les hypothèses (comme par exemple le transfert de bassin à bassin), on perçoit toutefois tout l'intérêt de l'application au Proche-Orient de tels principes ou de dispositions ou règles s'en inspirant, notamment concernant le bassin du Jourdain ou l'aquifère montagneux de Judée-Samarie, les pays et entités de la région négociant pour parvenir à un règlement fondé sur les principes de «communauté d'intérêt» et de "part raisonnable et équitable " dans le sens d'une gestion commune et de restriction de la consommation [44].

D'une manière plus pragmatique, l'échange de données et d'informations apparaît à tous les observateurs comme la condition préliminaire sine qua non d'une amorce de résolution du problème.

Ainsi par exemple, aucun des co-riverains du bassin du Jourdain et du Yarmouk n'a jamais jusqu'à présent échangé quelque information que ce soit, ni fait part aux autres riverains des travaux et ouvrages qu'il envisageait d'entreprendre ou qu'il y avait réalisés. De la même façon, le caractère fragmentaire, voire dans certains cas inexistant, des informations dont disposent les Palestiniens à propos des ressources en eau de la Cisjordanie constitue un obstacle majeur à une évaluation certaine de ces ressources [45]. En effet, les hydrologues palestiniens qui se sont à cet effet regroupés dans une association (le Groupe des Hydrologues Palestiniens) tentent vainement depuis des années d'obtenir de telles données [46].

La proposition de la constitution d'une banque de données régionales, fiables et accessibles a été faite par la Communauté européenne en mai 1992 à Vienne sur la base d'un rapport préparé par le B.R.G.M. à l'intention de la
Commission. Il s'agit d'y regrouper les données relatives aux ressources, tant sous leur aspect quantitatif que qualitatif (pluviométrie, infiltration, débits, recharges, qualité des eaux), aux prélèvements (en surface et dans les nappes souterraines) ainsi qu'aux principales structures de stockage et de régulation, à la distribution de l'eau et à son utilisation par secteur (réseaux de transport et de distribution, consommation humaine, agricole, industrielle, hydroélectricité, traitement et recyclage, dessalement, tarification)

Fin octobre de la même année, les ministres chargés de l'eau des Etats du bassin méditerranéen ont signé une Charte méditerranéenne de l'eau, agréée par la Communauté européenne en tant que bailleur de fonds, confirmant leur volonté commune d'oeuvrer au renforcement des structures et des moyens de mesure et de collecte des informations et données, à l'inventaire et à l'évaluation périodique des ressources disponibles, à la mise en place d'une banque de données sur les consommations et usages de l'eau, de plans de développement et schémas d'aménagement et de systèmes de tarification adéquats.

Cela a également constitué l'une des préoccupations majeures des signataires de la Déclaration de Rio qui recommande aux Etats la constitution de bases de données interactives, l'établissement de modèles de prévision et de planification économique ainsi que l'élaboration de méthodes de gestion et de planification des ressources en eau [47].

Dans l'immédiat, il devient donc plus qu'urgent de mettre en place une commission technique - tripartite dans un premier temps - de gestion de la ressource en eau, profitant de la dynamique des négociations menées, d'une part entre Israël et l'O.L.P., d'autre part entre Israël et la Jordanie.

Il s'agit en effet de promouvoir l'idée d'une communauté de l'eau «à géométrie variable " incluant dans un premier temps les pays et entités qui ont à faire face aux besoins les plus urgents, dans la mesure où ils ne disposent que de ressources hydrauliques et énergétiques limitées.

Une paix durable entre Israéliens, Palestiniens et Jordaniens suppose en effet que soit résolu le problème de leur approvisionnement stable et suffisant en eau et en énergie.

Dans un tel cadre, des réflexions et actions peuvent en particulier être menées sur le partage de la ressource en y intégrant si possible la Jordanie.

D’ores-et-déjà, des spécialistes israéliens proposent des solutions de partage, qu'il s'agisse de MM. ZAROUR et ISAAC déterminant au moyen d'une formule mathématique les parts respectives des utilisateurs [48] ou de Mme KLIOT suggérant un plan de répartition des eaux entre les différents protagonistes de la région [49].

\subsection{A moyen terme, la nécessaire mise en æuvre d'un système de gestion intégrée de la ressource en eau qui dépasse le seul cadre israélo-jordano- palestinien.}

Au-delà des solutions purement techniques susceptibles de contribuer partiellement à la résolution du problème tant au plan quantitatif (lutter contre le gaspillage implique la maîtrise de l'irrigation, le recyclage des eaux usées et la surveillance des réseaux ou le développement de procédés de substitutions tels que le dessalement de l'eau de mer) que qualitatif (lutte contre la pollution), il s'agit de sortir 
des situations de fait "priorité au premier occupant " et " priorité de l'utilisateur d'amont sur l'utilisateur d'aval ».

\subsubsection{Principes}

Pour ce faire et dans le cadre d'une communauté régionale de l'eau et de l'énergie du Proche-Orient susceptible à terme d'être élargie aux pays d'amont principaux détenteurs de la ressource (Turquie, Liban, Syrie, Egypte), on peut suggérer de substituer au monopole de l'autorité politique le principe d’une solidarité économique.

La nouvelle institution que l'on pourrait dénommer "Agence ou autorité régionale de l'eau pour le ProcheOrient (A.R.E.P.O. ou en anglais N.E.R.W.A. pour « NearEast Regional Water Agency ") - serait constituée sur le fondement des règles d'Helsinki (article 10) de "répartition raisonnable et équitable de l'eau dans le cadre d'un bassin international de drainage [50] " et en ce qui concerne les eaux souterraines transfrontalières selon les principes énoncés par le projet de traité de BELLAGIO, à savoir la gestion intégrée, la communauté d'intérêt, l'utilisation optimale et la préservation de la ressource sur une base raisonnable et équitable.

Dans un premier temps, cette institution pourrait être mise en place sous l'égide des Nations-Unies [51] mais à terme il importe que cette entité, non seulement soit directement gérée par les représentants des principaux pays "localement" intéressés mais y associe également des représentants des utilisateurs de l'eau voire même des élus de collectivités locales qui détiennent des compétences en matière d'eau, (adduction, distribution, assainissement).

Ceci implique dans une deuxième phase que les différents pays ou entités concernés acceptent de déléguer des compétences au profit d'une institution décentralisée et de lui conférer une large autonomie, conformément aux principes de décentralisation et de subsidiarité.

\subsubsection{Organisation générale et fonctionnement}

L'Agence régionale de l'eau pour le Proche-Orient comprendrait :

- une présidence tournante et un organe politique composé de représentants désignés par chacun des Etats ou entité pour une durée à déterminer :

- un conseil d'administration multinational tenant lieu d'exécutif, composé d'administrations, pour partie désignés par chaque pays ou entité, pour partie choisis par les membres du comité d'usagers (cf. infra) ;

- un comité d'usagers (organe délibérant) comprenant des représentants des différentes catégories d'utilisateurs (agriculteurs, industriels, consommateurs y compris des écologistes) et d'élus locaux choisis par leurs organisations professionnelles et politiques respectives :

- des observateurs d'autres pays susceptibles à terme de devenir membres de l'agence ;

- éventuellement, une commission spéciale chargée de prévenir et de résoudre les différends.

Le but recherché consiste, d'une manière pragmatique conciliant à la fois une approche par le sommet et par la base («top down/bottom up»), à mettre en cuvre une gestion intégrée de l'eau à l'échelon d'un bassin, d'un groupe de bassin ou d'un aquifère.
Il s'agit en même temps de susciter la confiance des bailleurs de fonds internationaux et c'est la raison pour laquelle une telle institution se doit d'être à la fois centralisée et décentralisée ainsi que dotée de compétences « intégrées ».

\subsubsection{Attributions et action à conduire}

L'agence s'attachera à gérer au mieux les antagonismes contenus dans les principes appliqués jusqu'alors de « priorité de l'amont sur l'aval " et de «priorité au premier occupant ». Son action sera dans un premier temps d'abord psychologique puisqu'elle devra tendre à faire disparaître l'idée selon laquelle ce qui constitue un droit pour les uns recouvre en fait un vol pour les autres...

L'objectif de l'opération est de :

- arbitrer et concilier les usages effectués par les différents pays ou entités au sein d'un forum spécialisé,

- donner à l'eau une valeur économique ;

- améliorer la disponibilité de la ressource tant au niveau quantitatif que qualitatif :

- éventuellement, procéder au partage de la ressource.

Bien évidemment, l'Agence disposera d'une banque de données constituée à partir d'informations mises en commun par les Etats-parties et selon les modalités précédemment évoquées.

L'Agence pourrait être habilitée à :

- construire et entretenir à titre de propriétaire les ouvrages importants de régulation et de soutien des étiages, ouvrages de traitement des eaux usées et de potabilisation (stations de traitement et de dessalement);

- dans un premier stade, impliquer les utilisateurs de l'eau dans les coûts (disponibilité de la ressource. épuration, potabilisation) afin d'être en mesure.

- dans un second stade de collecter des taxes incitant à moins consommer et à moins polluer l'eau de façon à pouvoir redistribuer les montants ainsi collectés sous forme de primes, subventions ou prêts aux utilisateurs qui consentent un effort pour accroître la disponibilité de la ressource ou diminuer les coûts pour l'Agence (par exemple, travaux de rationalisation des infrastructures comme l'irrigation, la réutilisation des eaux usées, la réfection des réseaux de distribution d'eau potable.

Le principe de base étant que l'eau ne constitue pas un bien gratuit et que chaque préleveur ou pollueur doit payer pour cet usage.

- Moduler le prix de l'eau selon le niveau de développement propre à la zone considérée, l'objet de l'utilisation (eau potable, irrigation, usage industriel).

Une telle proposition peut encore sembler, en l'état actuel des choses, ambitieuse voire même utopique. Toutefois, il est nécessaire d'anticiper une solution réelle et globale, en s'appuyant sur la dynamique de paix à l'œuvre au Proche-Orient qui s'est récemment concrétisée par l'accord israélo-palestinien du 26 octobre 1994 aux termes duquel Israël s'engage à laisser la Jordanie puiser 40 millions de mètres cubes par an dans le YARMOUK et à lui livrer 10 millions de mètres cubes à partir du lac de TIBÉRIADE, les deux Etats convenant par ailleurs de construire trois barrages (un sur le YARMOUK, deux sur le JOURDAIN) destinés notamment à l'approvisionnement en eau de la Jordanie à raison de 50 millions de mètres 
cubes/an. Pour prometteuses qu'elles soient, de telles avancées se doivent d'être gérées d'une façon intégrée.

En effet, l'émergence d'une zone de stabilité au ProcheOrient dépend pleinement d'une solution régionale et intégrée dans le domaine des ressources en eau, à laquelle ont intérêt y compris les pays les mieux pourvus dans ce domaine.

Partant du noyau israélo-palestinien, cette proposition peut constituer un schéma susceptible d'attirer graduellement d'autres pays, le moment venu, schéma dans lequel la France, forte de son savoir-faire dans le domaine de la planification et de la gestion intégrée des ressources en eau, pourrait être amenée à jouer un rôle.

En effet, l'expérience française des agences de l'eau fondée sur des mécanismes d'incitation économique à l'échelon du bassin (système de taxation des usages et d'aides concomitantes variant en fonction des efforts réalisés pour réduire la pollution ou la consommation de l'eau) ainsi que celle des compagnies d'aménagement en matière de gestion et de régulation d'ensembles complexes de ressources en eau (Compagnie d'aménagement des côteaux de Gasgogne, Société de Mise en Valeur de la Corse, Société du Canal de Provence etc...), seraient susceptibles d'apporter des éléments de réponse.

\section{Références}

[1] Cf. données du World resources Institute, « World Reserves 1992-1993: Towards Sustainable Development» OXFORD-NEW-YORK University Press 1992, p. 162.

[2] Mme FALKENMARK not. in «Global water issues confronting humanity ". Symposium sur l'eau dans les conflits armés, Comité international de la Croix-Rouge, Montreux, 21-23 nov. 1994.

[3] Depuis cette date, plusieurs sessions avaient eu lieu s'agissant des ressources en eau, respectivement à Vienne (1315 mai 1992), Washington (15-16 septembre 1992 et à Genève (28-29 avril 1993).

[4] M. Th. NAFF « Economic and socio-demographic factors of instability inn the Middle-East, and their relations to water issue " lors du «Southwest Asia and the Middle-East Strategy Development Seminar ", National Defence University's et Institute for National Strategic Studies 12-13 décembre 1989 éd. RA. COSSA et Ph. MARR, WASHINGTON, pp. 52 et s.

[5] M. Th. NAFF « Economic and socio-demographic factors of instability in the Middle-East, and their relations to water issue " lors du « Southwest Asia and the Middle-East Strategy Development Seminar », National Defence University's et Institute for National Strategic Studies 1213 décembre 1989 éd. RA. COSSA et Ph. MARR, WASHINGTON, pp. 52 et s.

[6] Chiffres cités par M. Christian CHESNOT « La bataille de l'eau au Proche-Orient " éd. L'HARMATTAN - Comprendre le Moyen-Orient 1993, p. 174.

[7] B.R.G.M. : Statistiques sur les ressources et les demandes en eau dans le monde (octobre 1989).

Tableau $\mathrm{n}^{\circ} 1$ C.EE.

[8] B.R.G.M. Etat des lieux du secteur de l'eau au MoyenOrient (juin 1992).

Tableau $n^{\circ} 2$ C.E.E.
[9] TAHAL (organisme responsable de la planification) « Water Master Plan, Report on the Water Sector in Israel, 1990 „ cité par Mme Natasha BESCHORNER « Water instability in the Middle-East » in ADELPHI, article 273, hiver 1992 1993 ,

éd. International Institute for Strategic Studies et BRAS SEY'S, p. 10.

[10] Capacité estimée entre 240 et 300 millions de $\mathrm{m}^{3} / \mathrm{an}$. Sur ces points, cf. MM. John BULLOCH et Adel DARWISH « Water Wars - Coming Conflicts in the Middle-East éd. Victor GollanCZ Londres 1993, pp. 43 et s.

[11] M. Th. NAFF et Mme Ruth C. MATSON \& Water in the Middle-East : conflict or cooperation ? " éd. BOULDER CO. WESTWIEW PRESS 1984, p. 231.

[12] Point de vue d'un hydraulicien israélien recueilli par M. Abdennour BENANTAR in « Le dossier hydraulique dans les négociations arabo-israéliennes : les risques d'une guerre pour l'eau " GLOBE vol. 1 n $^{\circ} 1$, printemps 1993, pp. 10-11.

[13] Contre 106 à l'industrie et 482 à l'usage domestique : chiffres du Bureau central des statistiques de I'Etat d'Israël (1991) cités par Mme BESCHORNER op. cit. (note 9), pp. $10 \mathrm{et} \mathrm{s}$

[14] «Libération" $n^{\circ} 3322$ du 26 janvier 1992, p. 22

[15] Op. cit. Mme BesChORNER, p. 11

[16] «Compagnie nationale des eaux" fondée en 1936 par l'Agence juive pour réaliser les projets hydrauliques en Palestine, constituée actuellement en société anonyme, l'Etat israélien détenant $33 \%$ des actions et le reste étant réparti entre l'Agence juive, le Fonds national juif et l'Union des travailleurs sionistes (cf. MM. ELDJERBAOUI et ABDELHADI « Les eaux de l'Etat de la Palestine : de la spoliation à la réappropriation ", in : Les Etudes Palestiniennes $n^{\circ} 4$, automne 1990 , p. 89 , cité par M. BENANTAR op. cit., pp. 2 et 8 )

[17] Sur les problèmes d'administration et de gestion de l'eau en Israël, cf. M. Richard LASTER, «ISRAEL » International Encyclopedia of Laws, avril 1993 ed. KLUWER DEVENTER/BOSTON, pp. 51 et s., 140-141 s'agissant en particulier de la pollution.

[18] MM. Joyce R. STARR et Daniel C. StolL « U.S. Foreign Policy on Water Resources in the Middle-East », Center for Strategic and International Studies (CSIS) Washington décembre 1987, pp. 12 et s.

[19] MM. Joyce R. StARR et Daniel C. StolL « US. Foreign Policy on Water Resources in the Middle-East » Center for Strategic and International Studies (CSIS) Washington déc. 1987, pp. 12 et $\mathrm{s}$.

[20] Seulement 12 millions pour M. Thomas NAFF in « Water Scarcity, Resource Management and Conflict in the MiddleEast, Environmental Dimensions of Security ", Proceedings from an Annual meeting symposium 9 fév. 1992, pp. 27 et 28. Cf. également la courbe prospective en annexe dressée par M. NAFF, tableaux 4 et 5 .

[21] Rapport du Conseil économique et social des Nations Unies 1992, cité par M. CHESNOT op. cit. note 6, p. 5.

[22] 775000 habitants, soit une densité de 3800 habitants au $\mathrm{km}^{2}$ selon les données du Haut Comité pour les Réfugiés des Nations Unies (Mars 1992).

[23] La quantité d'eau nécessaire à l'approvisionnement de l'enclave est estimée à 130 millions de $\mathrm{m}^{3} / \mathrm{an}$.

[24] La salinité de l'eau progresse chaque année de 15 à $20 \mathrm{mg} / \mathrm{l}$. et $70 \%$ des eaux souterraines dépassent le niveau de salinité de $500 \mathrm{mg} / 1$. (nomme guide O.M.S. $\max$. : $250 \mathrm{mg} / \mathrm{l}$ ). 
[25] M. ChesNot op. note 6, p. 175

[26] Pour le détail de ces plans, cf. MM. Hosh et ISAAC « Roots of the Water Conflict in the Middle-East ", Institut de recherche appliquée de Jérusalem, contribution présentée les 7-9 mai 1992 à l'Université de Waterloo (Canada) dans le cadre du « Symposium sur la crise de l'eau au Moyen-Orient - Perspectives et solutions ».

[27] Les installations de la centrale seront détruites par l'armée israélienne en 1948 pour éviter un contrôle des Arabes sur ces deux cours d'eau.

[28] Egalement détruit par les Israéliens en 1969.

[29] M. John K. COOLEY « The war over water " in : Foreign Policy $\mathrm{n}^{\circ} 54$, printemps 1984 , pp. 8-9.

[30] Cf. notamment M. BENANTAR op. cit. note 12, pp. 4 et $\mathrm{s}$.

[31] M. Benantar, op. cit. note 12, pp. 12-13. Cf. M. Hillet 1. ShUVAL \& Le problème du partage de l'eau entre Israël et les Palestiniens - A la recherche d'une solution équitable " Revue Monde-Arabe-Maghreb-Machrek $\mathrm{n}^{\circ} 138$, oct.-déc. 1992, pp. 34 et s. Egalement M. Walid ARBID « La stratégie de l'eau au Moyen-Orient »INCOM n 12, printemps 1994.

[32] Cité par Mme Beschorner op. cit. note 9, p. 13.

[33] Cf. M. CHESNOT op. cit. note 6, p. 177, le gouvernement israélien suventionne de façon très substantielle, les implantations juives par le biais de l'Organisation sioniste mondiale : de 15 à 23 agorot le $\mathrm{m}^{3}$ d'eau pour les colons juifs selon l'usage agricole ou domestique, 70 agorot pour les Palestiniens, quel que soit l'usage.

[34] Cf. MM. BULLOCH et DARWISH op. cit. note 1, p. 10, pp. 56-57.

[35] M. BENANTAR op. cit. note 12, pp. 8-9.

[36] M. Stephan LIBISZEWSKI « Die okologische Dimension des Nahost - Konfliktes» Weschselwirkung nr. 67 juin 1994 p. 13 et également du même auteur « Gerechte Wasserverteilung - ein Schlusselproblem in Nahen Osten " Tages Anzeiger 26 avril 1993, p. 2.

[37] Soit $590 \mathrm{de}^{3}$ pour l'irrigation et 490 pour la consommation domestique et l'industrie.

[37-1] M. Habib AYEB « Le bassin du Joudain dans le conflit israélo-arabe » Centre d'études et de Recherches sur le Moyen-Orient contemporain, Beyrouth-Amman-Cermoc 1993, p. 27

[38] MM. Eldierbaoui et Abdelhadi, cités par M. Benantar op. cit. note 12, pp. 14 et 15 .

[39] Le terme "Charia " lui-même serait dérivé d'un terme arabe signifiant l'eau. Sur ces points, ef. MM. Bulloch et DARWISH op. cit. note 10, pp. 160 et s. Le Coran enseigne qu'à partir de l'eau Dieu a créé tous les organismes vivants de l'univers.

[40] Règles d'Helsinki de 1966, International Law Association (I.L.A.), Report of the Fifty Second Conference, Londres 1969.
[41] Mme Patricia BuIRETTE "Genèse d'un droit fluvial international (utilisations à des fins autres que la navigation) " Revue générale de droit international public, janvier 1991, pp. 20 et s.

[42] Conférence des Nations Unies sur l'environnement et le développement (C.N.U.E.D.), "Action 21 », chapitre 18 pp. 142 et s., Nations Unies New-York 1993.

[43] Règles d'Helsinki, article VII.

[44] Transboundary Groundwaters: the Bellagio Draft Treaty C.I.R.T., éd. augmentée et revue par MM. R.D. HAYTON et A.E. UtTon, été 1989, Washington D.C. Voir également M.J. MARGat «Problèmes spécifiques aux nappes transfrontières " O.I.E. au /P.N.U.E. 28-30 avril 1992 Sophia-Antipolis.

[45] M. Khader Bichara « La géopolitique de l'eau en Méditerrannée " Centre d'Etudes et de Recherches sur le Monde Arabe Contemporain (C.E.R.M.A.C.). Université catholique de Louvain, 1994, pp. 17 et 19.

[46] Mme Nurit KLIOT « Building a legal regime for the JordanYarmouk River System - Lessons from other international rivers " (Université d'Haîfa) III $^{\mathrm{e}}$ conférence internationale de Durham. (Grande-bretagne) « La gestion pacifique des ressources transfrontalières » 14-17 avril 1994.

[47] M. CHESNOT op. cit. note 6, p. 181.

[48] « Action 21 », 18-12 op. cit. note 3 p. 30, p. 143.

[49] « Nature apportionment and the Cpen Market : a promising solution to the Arab-Israeli Conflict, Institut de recherche appliquée de Jérusalem, soit :

$\mathrm{A}(\mathrm{O})=50$

$\mathrm{A}(0)$ : Allocation de droits/obligation de l'état considéré (0) \%

$\mathrm{S}(0)$ : Superficie du bassin/volume de stockage en surface ou en souterrain dans les limites du territoire de l'état (0). $\mathrm{S}(\mathrm{T})$ : Superficie totale/volume de stockage du bassin. $\mathrm{V}(0)$ : Volume d' eau arrivant naturellement dans le bassin en provenance du territoire de l'Etat.

$\mathrm{V}(\mathrm{T})$ : Volume total d'eau arrivant dans le bassin.

$\mathrm{P}(0)$ : Volume de déperdition naturelle sur le territoire de l'état (0).

$\mathrm{P}(\mathrm{T})$ : Volume de déperdition naturelle pour l'ensemble du bassin.

[50] La notion de bassin international de drainage est définie comme étant une « zone géographique s'étendant a deux ou plusieurs Etats et déterminée par les limites de l'aire d'alimentation du système hydrographique, $y$ compris les eaux superficielles et souterraines, s'écoulant vers une embouchure commune" (Règles d'Helsinki, art. 2).

[51] En ce sens, M. CHESNOT op. cit. note 6, p. 219. 